acta medica

\title{
Head and Neck Radiology in AAV
}

Assoc. Prof. Elif Bulut, MD

ORCID: 0000-0003-0305-2239

ranulomatosis with polyangiitis (GPA), which is a rare antineutrophil cytoplasmic antibody (ANCA)

associated autoimmune disease, frequently (70\% to $100 \%)$ involves ear, nose and throat.

Imaging characteristics of sinonasal involvement are well described by computed tomography (CT) scans and includes mucosal thickening, osseous erosions or destructions and neoosteogenesis [1-4].

Although nodular thickening may suggest GPA, mucosal thickening is frequent but mostly a nonspesific finding that could be seen in various etiologies of sinusitis [4]. On the other hand, bony changes such as erosions/destructions and neoosteogenesis is highly suggestive of GPA, especially when clinical suspicion is high [1,2] (Figure 1).
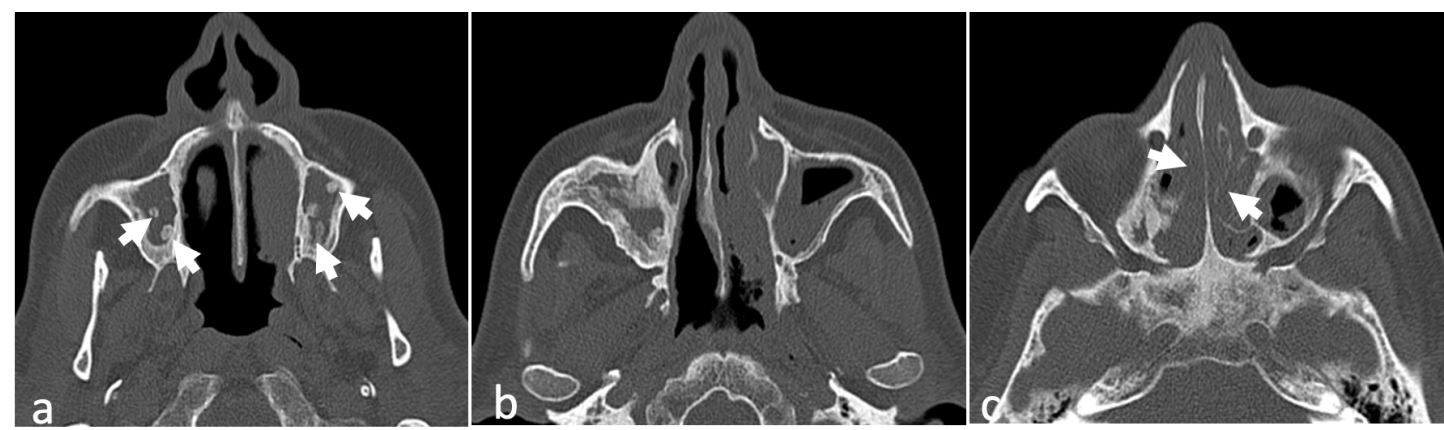

Note:

This manuscript has been peer-reviewed by Prof. Dr. Kader Karlı Oğuz before acceptance
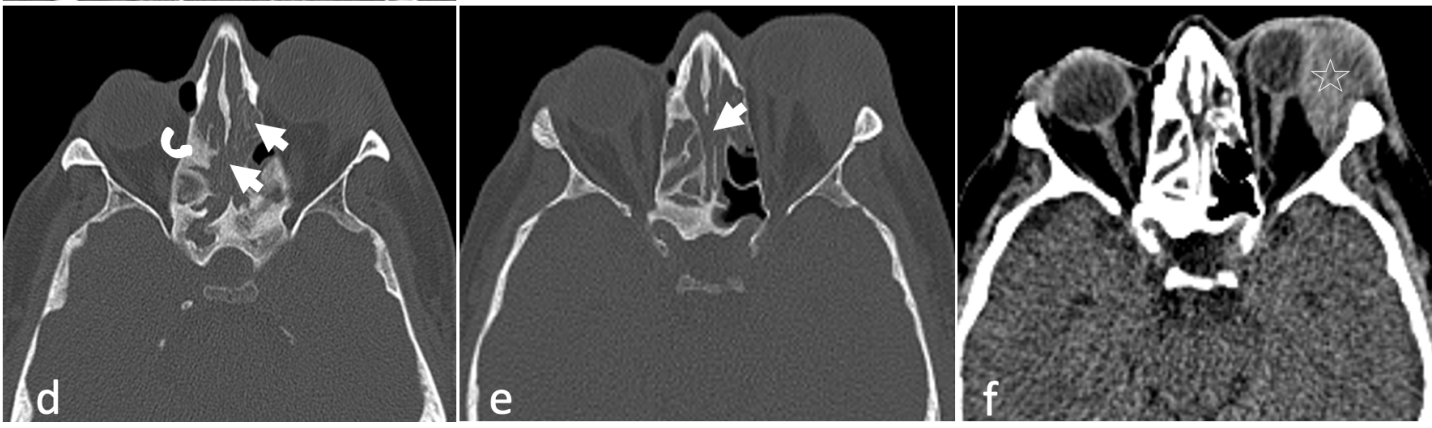

Figure 1: Axial CT images of fifty-three-year-old male patient with GPA. Bilateral maxillary sinuses have non-spesific opacifications and show atrophic changes. The walls of maxillary sinuses are thick and sclerotic due to chronic inflammation and have polypoid new bone formations inwards, representing neoosteogenesis (arrows in a). There are non-specific opacifications obliterating superior nasal cavity and ethmoidal cells causing erosions and focal bone defects at turbinates, ethmoid lamella and nasal septum (arrows in c, d, e). There are also accompanying sclerotic changes at ethmoid lamella (curved arrow in d). Please also note the orbital involvement manifested as a mass at lacrimal area (star) (f).

Bony erosions/destructions result from avascular necrosis due to granulomatous inflammation and fibrinoid necrosis of small to medium arteries. It frequently initiates from the midline structures and proceeds laterally to involve turbinates and paranasal sinuses [4], although cases sparing midline osseous structures could also be detected [5].

Neoosteogenesis has a typical imaging appearance and is characterized by a new bone formation inside the affected sinus wall. It particularly affects maxillary sinuses [2]. Neoosteogenesis is more frequently detected later in the course of the disease than in the disease onset. Accordingly, in the previous study enrolled in Hacettepe University Center of Vasculitis [5], neoosteogenesis were more frequent in the follow-up studies $(66 \%)$ than in studies at onset $(21 \%)$. In that study, osseous erosions were more common (40\%) in CT scans at disease onset than neoosteogenesis.

Hacettepe University Faculty of Medicine, Department of Radiology, Ankara
Besides paranasal sinuses, mucosal thickening could also be detected in nasal cavity [3]. İrregular mucosal thickening with ulcerations and septa in nasal cavity is relatively suggestive for the diagnosis of GPA [5]. 
Magnetic resonance imaging (MRI) is superior to CT imaging in revealing soft tissue changes associated with GPA. Due to the granulomatous nature of the disease, areas of involvement shows characteristically low T2 signal intensity and restricted diffusion (Figure 2). This feature could also be detected in fungal infection and other granulomatous diseases such as sarcoidosis. When osseous destruction and peri/ retroantral soft tissue changes are detected with typical MRI findings, imaging differentiation of GPA from invasive fungal sinusitis is hardly possible without a clinical guidance. The presence of other associated imaging findings whether at sinonasal location or other neighboring locations could help in differential diagnosis.
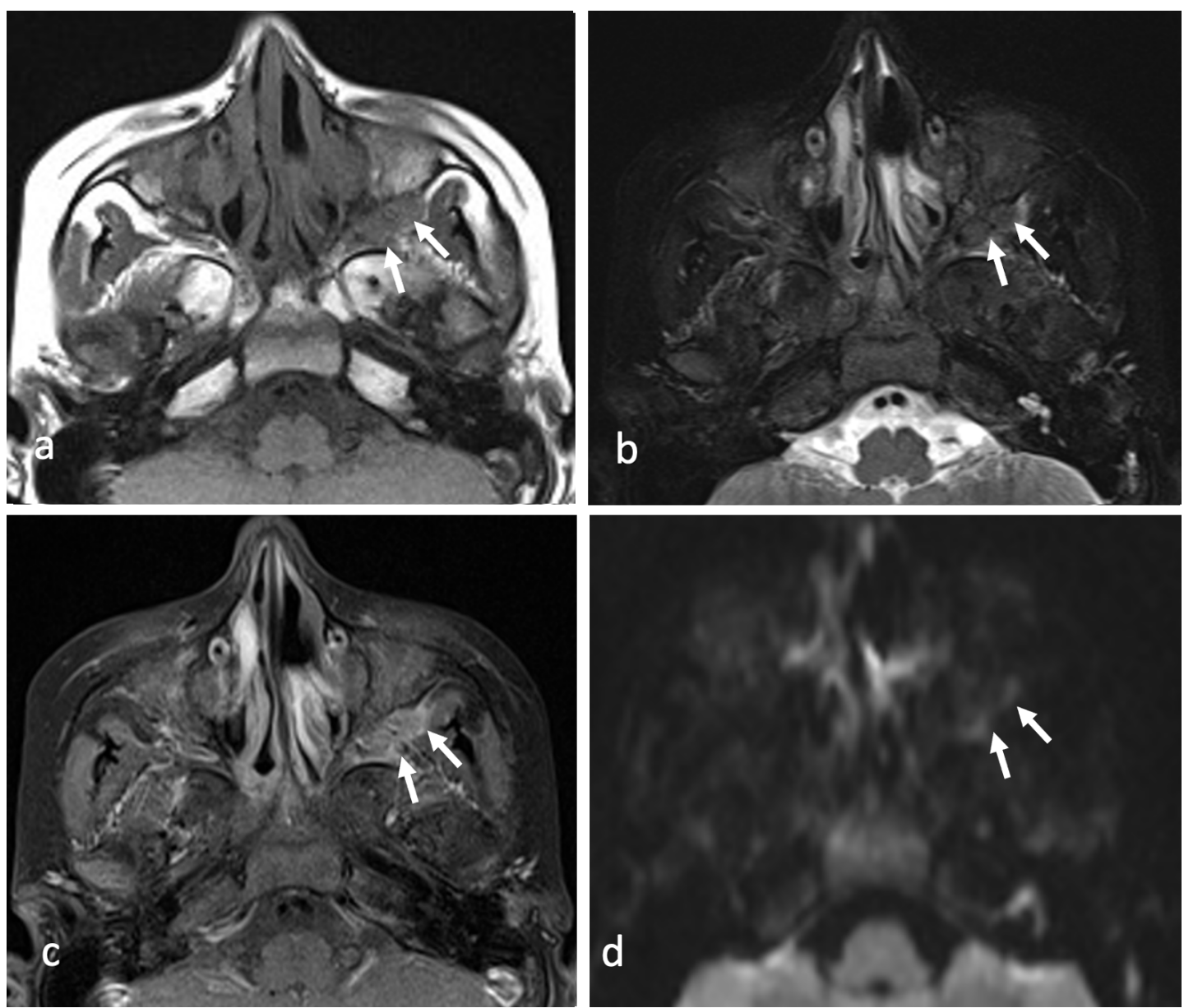

Figure 2: MR images of ten-year-old male patient with GPA. T1 weighted (W) axial image (a) shows hypointense soft tissue mass at retroantral area which is hypointense in T2W image (c), shows enhancement in fat saturated postcontrast T1W image (d), and restricted diffusion in diffusion weighed image (d).

\section{Orbital imaging findings of Granulomatosis with polyangiitis}

Orbital involvement is considered as one of the most frequent manifestations of GPA. It may be involved secondary to spread from sinonasal involvement or seen as an isolated involvement [6]. Data concerning imaging characteristic, especially MRI, are limited to few case reports [7-9]. The involvement could be bilateral or unilateral, mass like or infiltrative, and could affect lacrimal area, extraocular muscles and intraorbital fat planes. It is radiologically difficult to differentiate infiltrative pattern from idiopathic orbital inflammation, IgG4-related disease or lymphoma (Figure 3). 

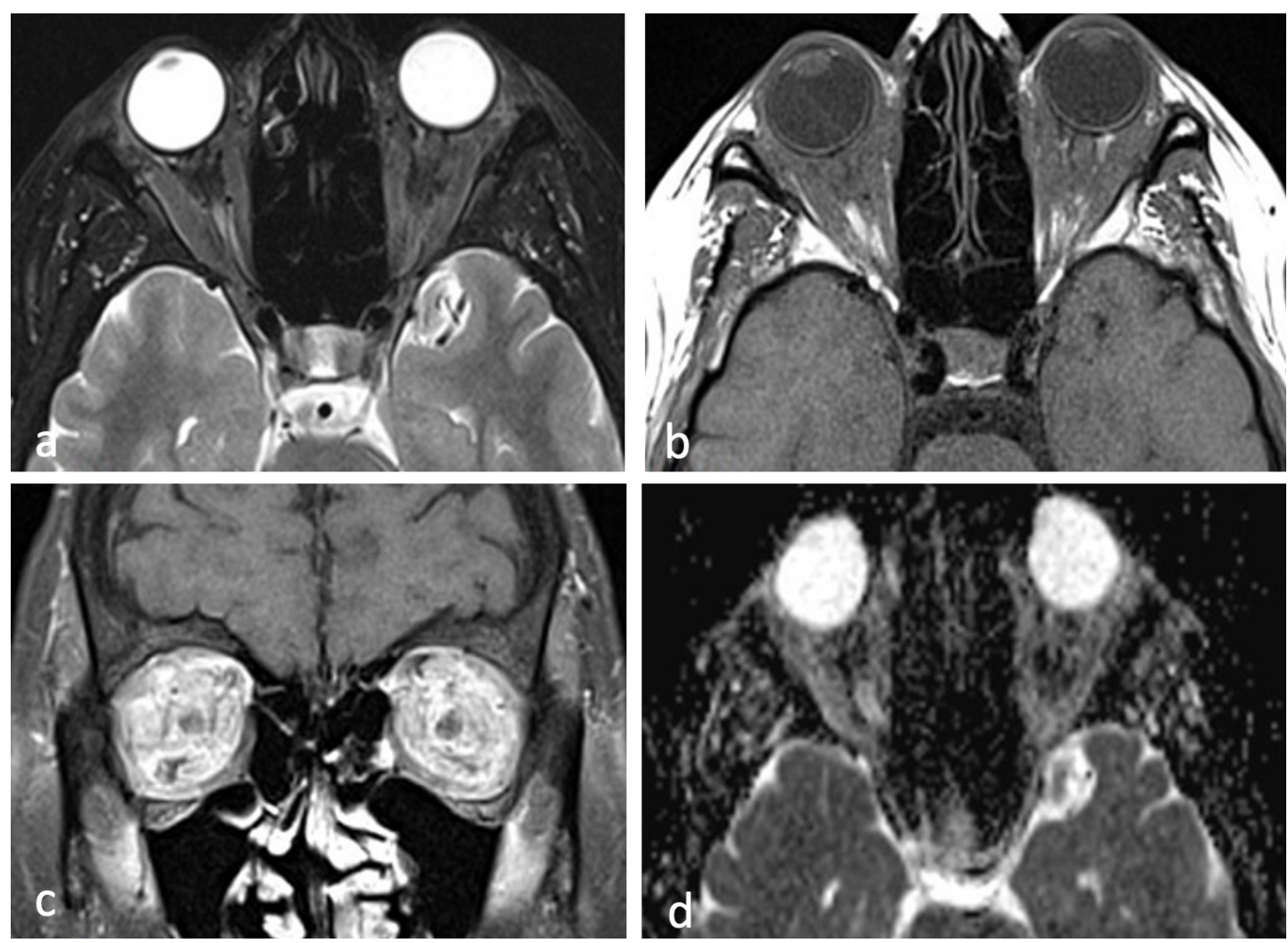

Figure 3: MR images of forty-seven-year-old female patient with GPA. Fat saturated axial T2W (a) and axial T1W (b) images demonstrate bilateral, infiltrative orbital involvement which shows enhancement at fat saturated coronal post-contrast image (c) and restricted diffusion at ADC maps.

\section{Nasopharyngeal and temporal imaging findings of Granulomatosis with polyangiitis}

The imaging characteristics of nasopharyngeal and temporal involvement are under-recognized compared to sinonasal manifestations. The radiologic data comes generally from single cases and small series of nasopharyngeal involvement mimicking nasopharyngeal carcinoma [10-12]. In the study enrolled in Hacettepe University Center of Vasculitis [5], nasopharyngeal involvement is revealed as not an infrequent finding; detected in $23 \%$ of the patients, particularly at onset. Only one of these patients had nasopharyngeal disease during follow-up. This is a remarkable finding and explains partly why the patients in previous case reports mostly misdiagnosed as nasopharyngeal carcinoma. Even more complicating the issue, the imaging findings of nasopharyngeal involvement are similar to nasopharyngeal carcinoma including asymmetric or symmetric soft tissue thickening with possible ulcerations, parapharyngeal infiltration and retropharyngeal lymphadenopathy (Figure 4). MRI characteristics are similar with the other ENT parts of involvement and characterized with low T2 signal intensity and restricted diffusion. Therefore, nasopharyngeal involvement of GPA could be challenging to differentiate not only from nasopharyngeal cancer, also from lymphoma or the other granulomatous inflam-matory diseases. Overlapping clinical and imag $\neg$ ing manifestations in nasopharyngeal involvement could cause diagnostic and eventually treatment delays. This underlines the role of future comparative studies to find a potential imaging finding that could discriminate nasopharyngeal involvement of GPA from mimicking pathologies.

Temporal involvement typically presents as serous otitis media caused by obstruction of the eustachian tube due to direct extension of adjacent sinonasal disease [1]. Therefore, most of the patients with temporal involvement have coexistent sinonasal and/or nasopharyngeal disease [5]. CT manifestations are non-specific and includes opacifications of the middle ears and mastoid cells with thickening of bony septa $[1,5]$. Granulomatous inflammation in middle ear and mastoid cells can also cause bone destruction mimicking coalescent otomastoiditis [1]. 

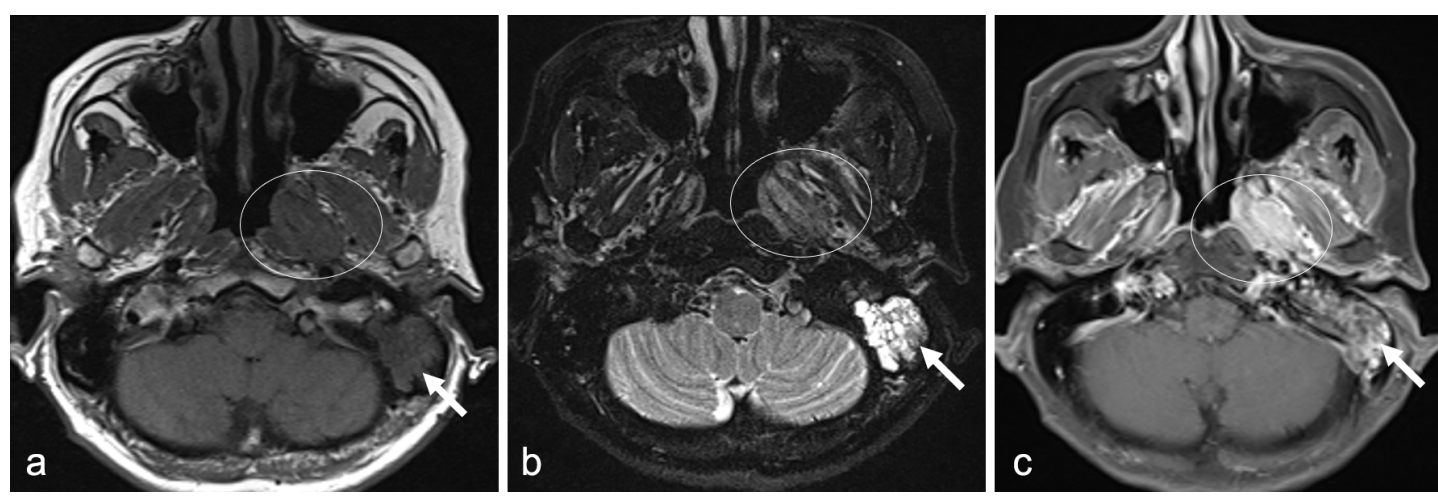

Figure 4: MR images of fifty-five-year-old female patient with GPA. T1W axial (a) and fat saturated axial T2W images (b) show soft tissue mass (circles) located at left nasopharynx wall with minimal parapharyngeal infiltration. The soft tissue mass shows diffuse enhancement in fat saturated post-contrast T1W image (c). Please also not associated inflammatory changes in left middle ear and mastoid cells (arrows).

\section{Laryngeal imaging findings of Granulomatosis with polyangiitis}

Laryngeal involvement of GPA is characterized with subglottic stenosis with circumferential thickening, irregularity and ulceration due to chronic mucosal inflammation and eventually fibrosis $[1,5]$ (Figure 5). It is defined as the most frequent airway manifestation and the frequency of this typically late manifestation of GPA ranges from $7 \%$ to $50 \%$ in studies $[2,5,13,14]$.

Figure 5: Axial CT image of forty-one-year-old female with GPA shows subglottic stenosis due to circumferential

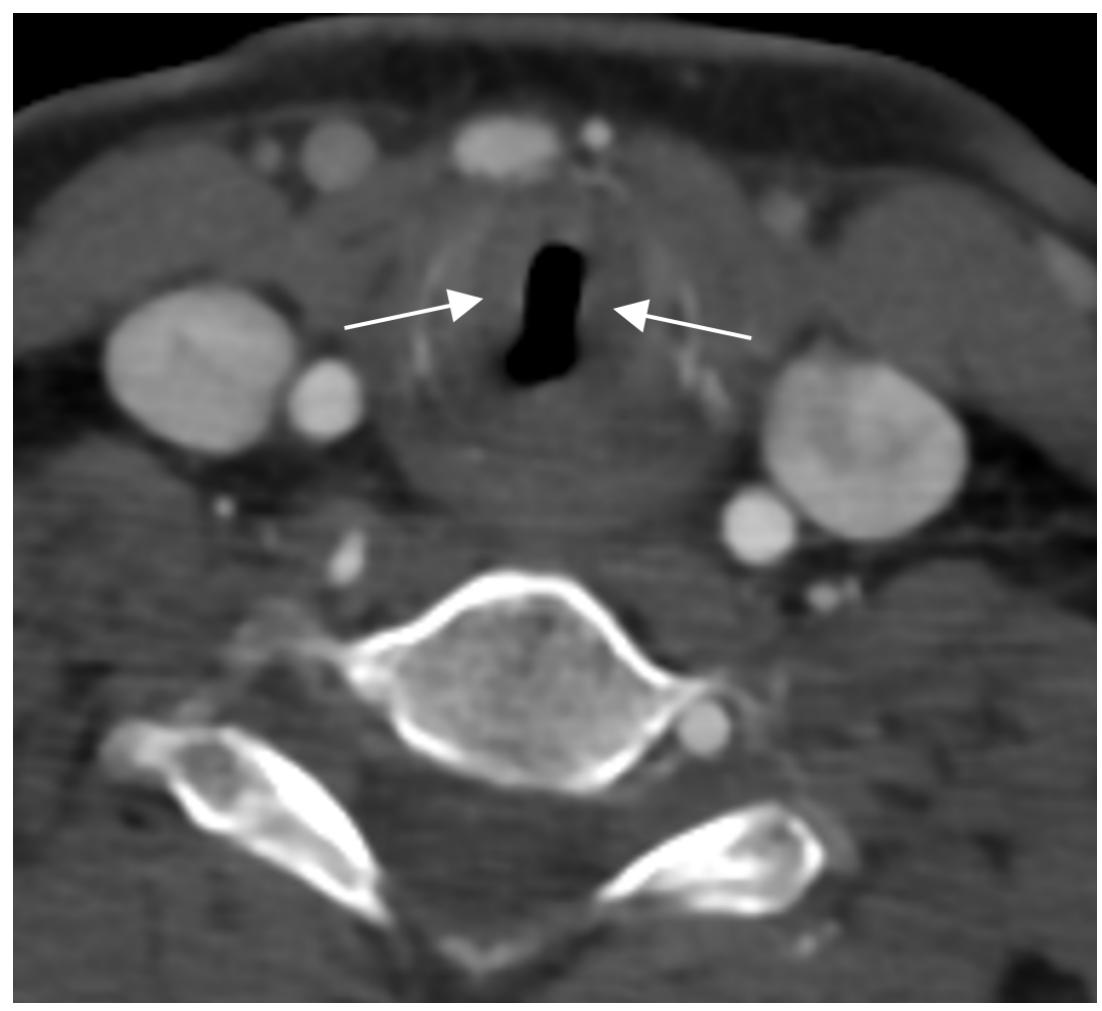

\section{Head and neck imaging in Eosinophilic granulomatosis with polyangiitis}

Eosinophilic granulomatosis with polyangiitis (EGPA), previously known as the Churg-Strauss syndrome (CSS) presents with sinusitis and sinonasal polyposis in the head and neck. Rarely, patients with in late stage EGPA may develop otitis media and sensorineural hearing loss [15]. CT imaging findings of patients with sinonasal polyposis include polyps occupying nasal cavity and paranasal sinuses and associated local bone remodeling or erosions (Figure 6). 


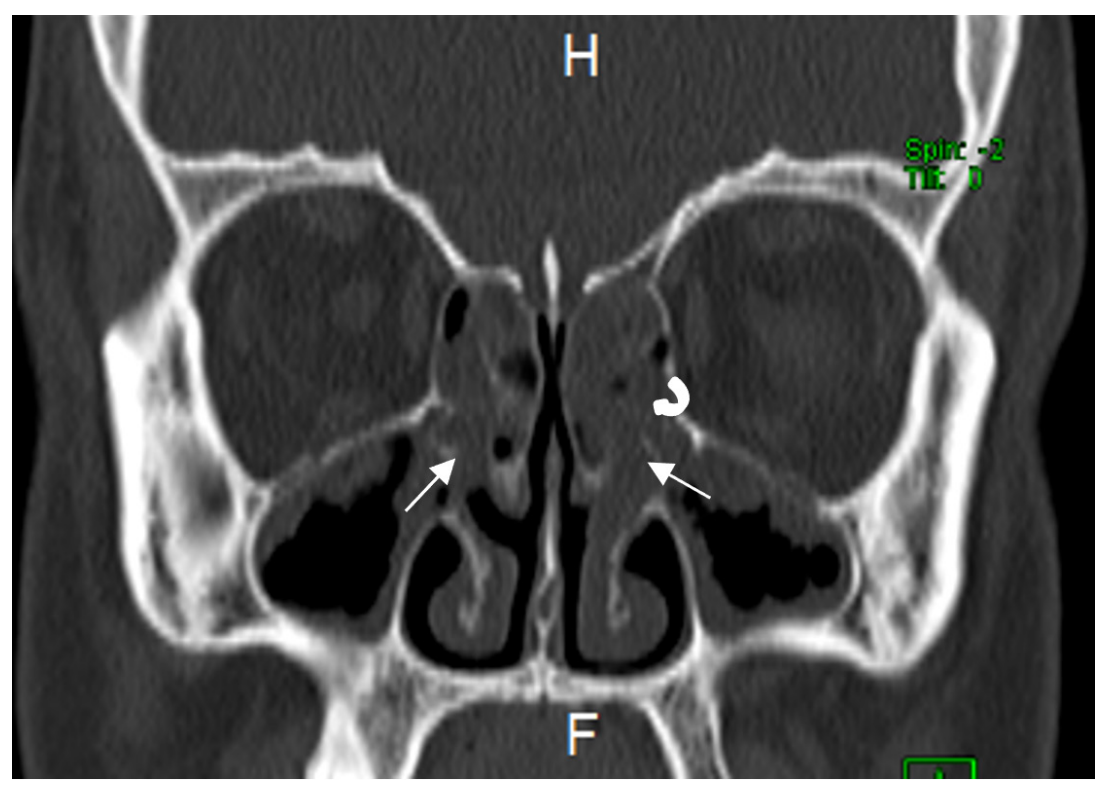

Figure 6: Coronal reformatted CT image of 29-year-old male patient with EGPA shows findings of sinonasal polyposis with polypoid opacifications in middle meatus (thin arrows) and ethmoid cells with erosive changes of ethmoid lamella (curved arrow). Also note peripheral nonspesific opacifications in maxillary sinuses.

ANCA associated vasculitis has varied manifestations in the head and neck. Imaging findings could overlap with other infectious-inflammatory diseases and malignancies. A detailed prior clinical information is essential for appropriate radiologic evaluation. The imaging findings that are more specific for these entities and diffusion imaging could help to narrow differential diagnosis.

\section{References}

1. Pakalniskis MG, Berg AD, Policeni BA, Gentry LR, Sato Y, Moritani T, et al. The Many Faces of Granulomatosis With Polyangiitis: A Review of the Head and Neck Imaging Manifestations. AJR Am J Roentgenol. 2015;205(6):W619-29.

2. Grindler D, Cannady S, Batra PS. Computed tomography findings in sinonasal Wegener's granulomatosis. Am J Rhinol Allergy. 2009;23(5):497-501.

3. Lohrmann C, Uhl M, Warnatz K, Kotter E, Ghanem N, Langer M. Sinonasal computed tomography in patients with Wegener's granulomatosis. J Comput Assist Tomogr. 2006;30(1):122-5.

4. Benoudiba F, Marsot-Dupuch K, Rabia MH, Cabanne J, Bobin S, Lasjaunias P. Sinonasal Wegener's granulomatosis: CT characteristics. Neuroradiology. 2003;45(2):95-9.

5. Bulut E. Granulomatosis with polyangiitis: Special focus on suggestive imaging findings in head and neck involvement. Acta Medica. 2019;50:9-16.

6. Sfiniadaki E, Tsiara I, Theodossiadis P, Chatziralli I. Ocular Manifestations of Granulomatosis with Polyangiitis: A Review of the Literature. Ophthalmol Ther. 2019;8(2):227-34.

7. Drobysheva A, Fuller J, Pfeifer CM, Rakheja D. Orbital Granulomatosis With Polyangiitis Mimicking IgG4-Related Disease in a 12-Year-Old Male. Int J Surg Pathol. 2018;26(5):453-8.

8. Yang B, Yin Z, Chen S, Yuan F, Zhao W, Yang Y. Imaging diagnosis of orbital Wegener granulomatosis: A rare case report. Medicine (Baltimore). 2017;96(23):e6904.

9. Takazawa T, Ikeda K, Nagaoka T, Hirayama T, Yamamoto T, Yanagihashi M, et al. Wegener granulomatosis-associated optic perineuritis. Orbit. 2014;33(1):13-6.

10. Helmberger RC, Mancuso AA. Wegener granulomatosis of the eustachian tube and skull base mimicking a malignant tumor. AJNR Am J Neuroradiol. 1996;17(9):1785-90.

11. La Rosa C, Emmanuele C, Tranchina MG, Ippolito M, Cosentino S, Saita V, et al. Diagnostic consideration for sinonasal Wegener's granulomatosis clinically mistaken for carcinoma. Case Rep Otolaryngol. 2013;2013:839451.

12. Mohapatra A, Holekamp TF, Diaz JA, Zebala L, Brasington R. Atlantoaxial subluxation and nasopharyngeal necrosis complicating suspected granulomatosis with polyangiitis. J Clin Rheumatol. 2015;21(3):156-9.

13. Kuhn D, Hospowsky C, Both M, Hey M, Laudien M. Manifestation of granulomatosis with polyangiitis in head and neck. Clin Exp Rheumatol. 2018;36 Suppl 111(2):78-84.

14. Polychronopoulos VS, Prakash UB, Golbin JM, Edell ES, Specks U. Airway involvement in Wegener's granulomatosis. Rheum Dis Clin North Am. 2007;33(4):755-75, vi.

15. Nwawka OK, Nadgir R, Fujita A, Sakai O. Granulomatous disease in the head and neck: developing a differential diagnosis. Radiographics. 2014;34(5):1240-56. 